\title{
Mechanical behavior of hybrid zirconia developed through Room Temperature Atomic Layer Deposition (RT-ALD)
}

Objectives: The aim Evaluate the mechanical performance of the hybrid interface between the transformed zirconia layer and the silica-based nanofilm, deposited by means of Atomic Layer Deposition at Room Temperature (RT-ALD) as well as the bond strength between the zirconia and the resin cement after RT-ALD technique.

Materials and Methods: Electron Microscopy (TEM)/Scanning Electron Microscopy (SEM), Energy Dispersive X-ray Spectroscopy (EDX), X-ray Diffraction (XDR) and Fourier Transform Infrared (FTIR). Fully-sintered Y-PSZ specimens in different translucencies (MO, MT, LT) were distributed in 5 groups: control (C - no treatment); hydrothermal treatment (HT- $15 \mathrm{~h}-134^{\circ} \mathrm{C}, 2 \mathrm{bar}$ ); alumina blasting (B - $50 \mu \mathrm{m} \mathrm{Al}_{2} \mathrm{O}_{3}$ ); RT-ALD silica deposition (S); HT followed by silica deposition (HTS). RT-ALD cycles consisted of the sequential exposure of specimens to tetramethoxysilane orthosilicate (TMOS - 60s) and ammonium hydroxide $\left(\mathrm{NH}_{4} \mathrm{OH}-10 \mathrm{~min}\right.$ ) vapors in 40 cycles. Mechanical performance was analyzed by flexural strength (FS) and fatigue failure load tests. Surface hardness (H) and Young's modulus (YM) were analyzed by nanoindentation. For surface chemical and topographical characterization, X-ray photoelectron spectroscopy (XPS) and scanning electron microscopy (SEM) were performed. For the shear bond strength test (SBS), composite resin cylinders were cemented on the zirconia surface with resin cement (Multilink Automix) and, after the test, the failure mode was evaluated. Data from surface $\mathrm{H}$, YM, FS, SBS and fatigue limit (FL) were analyzed by two-way analysis of variance (ANOVA).

Results: The surface topography was rougher for the blasted groups. In the XPS analysis, a silica nanofilm was observed on the zirconia surface after RT-ALD. Bond strength values of group S and HTS were similar to group J ( $p>0.848)$. Both $S$ treatments showed values of FS similar to groups B $(p>0.410)$. S did not affect FL when compared to group C $(p>0.277)$ for all evaluated materials.

Conclusions: RT-ALD technique was effective in depositing silica on the zirconia surface, presenting results of bond strength similar to the blasted specimens. In addition, RT-ALD did not have any deleterious effect on the mechanical properties. 